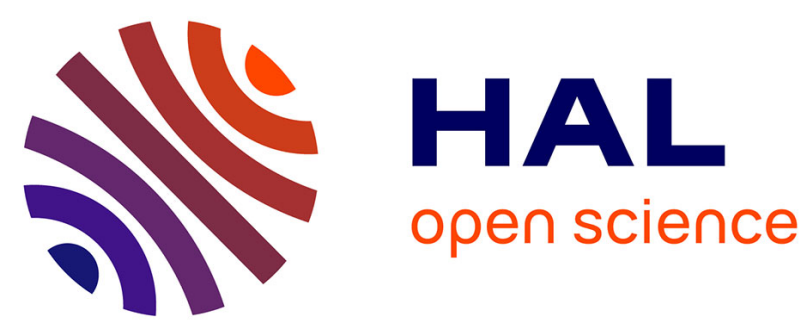

\title{
Vers un accompagnement sociobiographique contextualisé du français langue d'intégration en Guadeloupe : retour d'expérience
}

Frédéric Beaubrun

\section{- To cite this version:}

Frédéric Beaubrun. Vers un accompagnement sociobiographique contextualisé du français langue d'intégration en Guadeloupe: retour d'expérience. Contextes et Didactiques, 2016, Accompagnement sociobiographique en contexte post-colonial: Plurilinguisme, Émancipation, Formation, 8, pp.65-63. hal-01689080

\section{HAL Id: hal-01689080 \\ https://hal.univ-antilles.fr/hal-01689080}

Submitted on 20 Jan 2018

HAL is a multi-disciplinary open access archive for the deposit and dissemination of scientific research documents, whether they are published or not. The documents may come from teaching and research institutions in France or abroad, or from public or private research centers.
L'archive ouverte pluridisciplinaire HAL, est destinée au dépôt et à la diffusion de documents scientifiques de niveau recherche, publiés ou non, émanant des établissements d'enseignement et de recherche français ou étrangers, des laboratoires publics ou privés. 


\title{
Vers un accompagnement sociobiographique contextualisé du français langue d'intégration en Guadeloupe : retour d'expérience
}

\author{
Frédéric BEAUBRUN \\ Université des Antilles - CRREF (EA-4538)
}

\section{Résumé :}

Depuis 2011, l'État français a renforcé sa politique linguistique à l'égard des migrants en introduisant un dispositif de Français, langue d'intégration. Cette formation est destinée aux migrants souhaitant obtenir un permis de séjour sur le territoire français. Cet article présente deux sessions d'enseignement du français comme langue d'intégration selon une approche qui utilise des outils pédagogiques sociobiographiques. Nous nous penchons sur certaines sessions particulières en vue d'identifier des compétences linguistiques susceptibles d'être utilisées dans un programme d'enseignement du français. De cette façon, l'enseignant doit prendre soin des spécificités et des réalités contextuelles concernant la situation post-coloniale de la Guadeloupe. Il va choisir des moyens multimodaux pour aider les migrants à s'approprier leur nouvelle identité. L'analyse des fiches pédagogiques des enseignants, des productions des apprenants, des traces laissées au tableau et des transcriptions des interactions a permis de mettre en évidence la possibilité d'atteindre les objectifs d'appropriation linguistique tout en mettant en œuvre un accompagnement sociobiographique. Nous proposons ensuite des idées pour penser un système d'intégration linguistique en tenant compte du contexte et de l'histoire des apprenants pour y inclure leurs particularités propres et personnelles.

\section{Mots-clés :}

Français langue d'intégration, migrant, Guadeloupe, politique linguistique, accompagnement sociobiographique.

\begin{abstract}
:
Since 2011, the French State has reinforced its linguistic policy towards migrants by introducing a system of French as language of integration. This training is intended for migrants wishing to obtain a residence permit on French territory. This article presents two sessions of teaching French as a language of integration according to a new approach that uses socio-biographical pedagogic tools. We look at specific sessions to identify language skills that could be used in a French language teaching program. In that way, the teacher must take care of the specificities and contextual realities concerning the post-colonial situation in Guadeloupe. He shall choose multimodal means to help the migrants appropriate their new identity. The analysis of teachers' pedagogical worksheets, learners' productions, traces left on the board and transcriptions of interactions made it possible to highlight the possibility of achieving the objective of linguistic appropriation while using socio-biographical accompaniment. Then, we suggest some ideas to think a system of linguistic integration taking into account the context and the history of the learners in order to include their own and personnal peculiarities.
\end{abstract}

\section{Keywords :}

French language of integration, migrants, Guadeloupe, sociobiographical accompaniment, language policy. 


\section{Introduction}

Cette contribution vise à décrire, analyser puis discuter la mise en œuvre de deux séances pédagogiques d'enseignement du français langue d'intégration (désormais FLI) à destination d'un public allophone en Guadeloupe où le formateur a utilisé des stratégies particulières d'accompagnement sociobiographique. La Guadeloupe est une région monodépartementale française située dans la Caraïbe, et plus précisément dans les Petites Antilles. Elle présente un contexte sociolinguistique particulier avec deux langues en présence, le français et le créole guadeloupéen. Autrefois qualifié de diglossique, la situation sociolinguistique a évolué vers une pratique plus complexe où les locuteurs passent régulièrement d'une langue à l'autre, à l'intérieur d'une même phrase, ou d'une phrase à l'autre à l'intérieur d'un discours. Ces pratiques de contact de langues sont appelées alternances et mélanges codiques (Anciaux, 2013) entre langue normée et langue informelle, fluctuante et improvisée, à l'instar d'une situation observée au Québec dans les interactions entre l'anglais et le français où : " il ne s'agit pas de retraduire dans une deuxième langue ce qui vient d'être dit dans la première, c'est une seule et même pensée qui s'exprime en continu, il faut trouver un nom pour ce bilinguisme particulier : la biglossie » (Chevrier, 2013). Cette spécificité est aussi évoquée dans l'étude du langage des jeunes à la Réunion: "les jeunes Salaziens s'inscrivaient dans une "diglossie » classique ou dans une " bi-polyglossie » plus moderne » (Ledegen, 2002). Ainsi, soit les locuteurs opposent de façon diamétrale le créole et le français dans leur répartition fonctionnelle et complémentaire (situation informelle versus formelle), soit les biplurilingues vivent de façon moins stricte la pratique des deux langues en présence et manient le mélange des langues de façon ludique, pragmatique et identitaire.

Dans ce contexte sociolinguistique particulier, la Guadeloupe accueille principalement des populations de migrants issus de pays de la Caraïbe, tels qu'Haïti, la République Dominicaine et la Dominique, mais aussi d'autres régions du monde, comme la Syrie et la Chine. Au niveau administratif, ces personnes doivent justifier de leur maîtrise de la langue française afin de pouvoir résider sur le territoire. Elles doivent passer un test d'évaluation de français, et celles qui présentent des difficultés doivent suivre des formations de FLI dans des centres habilités depuis 2011. C'est dans ce cadre et ce contexte que notre recherche a été menée. La problématique est de savoir si la formation pour adultes dans cet espace culturel défini peut utiliser des approches sociobiographiques afin de favoriser les processus pédagogiques. L'évocation de l'histoire de vie des apprenants dans un dispositif de FLI peut-elle être un outil facilitateur de leur processus d'intégration tout en développant leur maîtrise de la langue française ? La verbalisation des expériences et la reconnaissance de leur bagage culturel et linguistique peuvent-elles être réinvesties dans leur intégration linguistique et sécuriser leurs apprentissages ?

L'introduction d'un accompagnement sociobiographique en FLI vise la transmission des savoirs linguistiques aux migrants en s'appuyant sur les langues en présence et sur leurs histoires de vie. Cette démarche cherche à faire advenir la connaissance à partir du « déjà-là » langagier et expérientiel des participants. Il s'agit d'une posture maïeutique et d'un choix didactique basé sur le concept de phénoménologie décrit par Romano (2010) et repris dans les travaux de Castellotti (2015) où l'objectif est de "réintroduire l'être dans son entièreté et dans son expérience » (Castellotti, 2015 : 249). Les relations entre l'apprenant et le formateur et celles entre les apprenants deviennent source d'appropriation. Cette posture pédagogique rejoint celle qui est analysée par Molinié (2006) dans son aspect sociobiographique où la dynamique introspective de l'apprenant par un retour réflexif à sa propre histoire de vie constitue une force pour l'apprentissage. Ainsi, par une mobilisation réflexive des expériences 
de vie, la transposition didactique n'est plus seulement basée sur la transformation de savoirs savants en savoirs à enseigner (Chevallard, 1986), mais aussi sur les prédispositions affectives, culturelles et relationnelles des apprenants afin de les confronter à un ensemble de prescriptions langagières. Le processus d'appropriation opère par la voie d'une conjugaison des différences de chacun. Il doit permettre le dépassement des tensions dues à la présence d'une langue française en position dominante et hégémonique dans un environnement postcolonial, plurilingue et multiculturel. Cette approche basée sur l'ipséité des apprenants vise leur émancipation. La posture pédagogique mise en œuvre accepte une part d'instabilité calculée et régulée. Le principe du risque d'incertitude pédagogique y est utilisé. La démarche est décrite sous l'angle de l'appropriation, processus plus instable que ceux d'acquisition et d'apprentissage (Castellotti, 2015 ; Besse et Porquier, 1991 ; Porquier et Py, 2004). Nous tentons de formuler des propositions qui "permettent d'entrer dans l'intelligence de la culture de l'autre » (Meirieu, 2014).

Nous sommes impliqué à deux niveaux dans le travail présenté dans cette contribution, d'une part en tant que formateur en FLI, et d'autre part comme chercheur en éducation. Nous avons conçu, réalisé et animé les séances de cette expérimentation. Ce travail s'inscrit dans le cadre d'une thèse de doctorat en Sciences de l'Education menée à l'université des Antilles. Elle vise à décrire les enjeux du FLI, comprendre les stratégies des formateurs et les attentes des apprenants, et proposer des pistes de réflexion et des perspectives didactiques contextualisées en Guadeloupe. Par la suite, nous présentons notre expérimentation en définissant les caractéristiques de notre population d'étude, l'articulation de l'action au cadre théorique, le déroulement de la formation et le protocole de recueil des données. Nous exposons et discutons ensuite les principaux résultats obtenus.

\section{Présentation de l'action de formation}

\subsection{Présentation du public et des objectifs}

Le public concerné est composé de 20 participants, dont 1 homme et 19 femmes. Ils ont répondu à une proposition de formation gratuite. Tous étaient volontaires pour participer à cette action. La majorité des personnes sont hispanophones et originaires de la République Dominicaine, sauf deux d'entre elles en provenance d'Haïti. La plus jeune est âgée de 20 ans et la plus âgée de 64 ans. La moyenne d'âge est de 40 ans. La moitié des apprenants avait suivi un cursus scolaire au moins jusqu'au lycée dans leur pays d'origine et l'autre moitié jusqu'au collège. Les participants ne connaissaient que quelques mots de créole au départ, sauf les deux haïtiennes, et ont intégré cette langue progressivement aux côtés du français pendant la formation. La plupart des participants était sans emploi, sauf cinq personnes dont deux étaient scolarisées au lycée, une qui travaillait dans une bibliothèque et deux au consulat de la République Dominicaine. Seulement un tiers des participants est passé auparavant par un cursus habituel en FLI. Chez cette population migrante, nous avons repéré un phénomène récurrent d'insécurité linguistique chez les migrants concernés due à plusieurs facteurs conjugués. La situation dichotomique de diglossie (Fergusson, 1959), héritée du système esclavagiste aux Antilles, met en relations une langue dite "haute », l'acrolecte français, prestigieuse et utilisée lors des contacts avec les administrations et dans le système scolaire officiel avec une langue dite "basse », basilecte créole, pratiquée au quotidien lors des échanges informels ou amicaux. Ceci oblige le migrant à se repérer par rapport à la nature de chaque situation rencontrée. Le locuteur se trouve alors devant une obligation de choisir parmi plusieurs formes d'expression et " il peut y avoir une relation directe entre contexte de minoration de langues et émergence d'un sentiment d'insécurité » (Jeannot-Fourcaud, 2016 : 
81). Le migrant peut se retrouver dans une situation dite de "schizoglossie" face au "conflit qui se pose (...) lorsqu'il doute de ce qu'il doit dire ou écrire parce qu'un même "item" linguistique se présente à lui sous plus d'une forme » (Haugen, 1962, dans Jeannot-Fourcaud, 2016). L'insécurité est augmentée à cause du caractère centralisateur des prescriptions du FLI fondées sur la matrice d'un programme national avec des supports d'examens souvent hors contexte (images de trains et gare SNCF). La France métropolitaine est prescriptrice de normes et de référentiels qui nous conduisent à parler d'un «conflit oedipien identitaire caribéen » (Beaubrun, $2014: 59$ ) que nous définirons comme une difficulté à construire son identité à cause d'un conflit de loyauté ${ }^{1}$ et d'une situation de glottophobie (Blanchet, 2016). Le migrant est pris en étau entre le besoin d'être accepté par le dominant, quitte à l'imiter et son besoin de définir et de défendre sa culture insulaire et son identité notamment linguistique. Doit-il seulement reproduire et transposer des normes ou au contraire créer ses propres outils d'intégration au monde? «Pour l'Europe, pour nous-mêmes et pour l'humanité, camarade, il faut faire peau neuve, développer une pensée neuve " (Fanon, 1961 : 305). Cette considération a guidé la mise en place d'objectifs spécifiques dans la formation que nous présentons.

Dans le programme de formation, nous avons privilégié certains objectifs fonctionnels et opérationnels: acquérir et/ou se réapproprier les savoirs de bases nécessaires pour la communication avec les administrations françaises, se présenter aux épreuves de certification du niveau de langue française avec plus d'assurance, se projeter dans une démarche d'intégration par l'activité, la formation et l'expression culturelle, s'exprimer tant à l'oral qu'à l'écrit, résoudre un problème quotidien en utilisant les phrases outils génériques appropriées, mieux se connaître pour clarifier ses choix professionnels et personnels, restaurer une image de soi, comprendre les possibilités de partage culturel et du vivre ensemble, valoriser sa culture d'origine et apprécier la culture du pays d'accueil. En vu d'atteindre ces objectifs, une stratégie d'enseignement a été définie. Celle-ci passe par la médiation culturelle afin de puiser dans des représentations communes aux diverses cultures en présence. Cette médiation utilisera des contes et des fables comme supports pédagogiques et moyen de se raconter en passant par l'imaginaire. Ceci est adossé à la volonté de faire correspondre la morale évoquée en fin de fables avec les règles édictées dans le référentiel FLI des valeurs de la République. Le contexte plurilingue y sera force de création d'outils d'expression. Les participants se verront ainsi offrir la possibilité de conceptualiser dans leur représentation symbolique du monde une cartographie de leur vécu menant à une stratégie de discours d'adaptation. Cette dynamique pourra œuvrer dans le sens d'un rééquilibrage des tensions du diptyque dominantdominé qui persiste en contexte postcolonial caribéen.

\footnotetext{
${ }^{1}$ D'un côté une loyauté à la langue française qui est synonyme d'acceptation dans la communauté du dominant, fidélité pragmatique, et d'un autre côté, une loyauté à la langue créole née du mélange des langues africaines et européennes à l'époque de l'esclavage, fidélité de cœur. Cette dualité crée une ambivalence linguistique aux Antilles et marque de façon masquée les intentions de communication entre les individus. De là peut provenir une des explications de la stigmatisation de la langue créole qui se trouve minorée et discriminée. Comme dans l'œdipe, reconnaitre l'importance de l'usage de la langue créole aux Antilles revient à tuer symboliquement la langue française du père, (la Métropole), pour s'approprier celle de la mère, (l'Afrique). C'est une étape qui doit mener, comme dans l'œdipe, à se construire une identité métissée, autonome et assumée, constituée du mélange des apports entre deux entités : cultures et langues. Ainsi nous observons un phénomène de discrimination des locuteurs uniquement créolophones quand ils ne maîtrisent pas également la langue française. A fortiori, le bagage linguistique des migrants apparait incomplet dans cet environnement sociolinguistique complexe où deux langues, le français et le créole, sont constitutives d'une compétence nécessaire à la communication et à la reconnaissance par les pairs.
} 


\subsection{Articulation de l'action aux contextes}

Afin d'articuler notre action de formation aux contextes, deux dimensions ont été envisagées. La première concerne le contexte sociolinguistique (contexte externe) et la seconde la dimension personnelle et sociobiographique des apprenants (contexte interne). L'objectif officiel du FLI est de s'approprier une "langue d'usage pratique, dont l'apprentissage se fonde sur des références quotidiennes, destinée à devenir la langue courante des apprenants, une langue familière » et «une langue d'autonomie qui permet à l'apprenant de se mouvoir dans les différents espaces de la société...» (Vicher, 2011). Il est apparu nécessaire d'articuler l'action de formation à la réalité sociolinguistique de la Guadeloupe en s'appuyant sur une pédagogie de la variation (Prudent, 2005) au regard de la présence de formes interlectales dans les pratiques langagières (Prudent, 1981). Nous proposons, à la suite de Romani concernant l'ile voisine de la Martinique (Romani, 2000), une démarche "qui parte de la production réelle et non de modèle à imiter" afin de "fonder une pédagogie du français et du créole sur cette zone interlectale qui est au cour de la langue maternelle " pour «faire acquérir à l'apprenant la maîtrise du système de variation (Georger, 2006 : 138). Ainsi la pédagogie choisie est basée sur l'articulation des langues en présence et une expression spontanée déculpabilisée en vue d'apprendre le français, car nous avons pu constater dans les interactions avec formateur lors des périodes d'interclasses, que les migrants reprennent presque systématiquement l'usage de leur langue d'origine. Il fallait donc probablement prendre en compte un besoin de s'exprimer en langue première et choisir d'intégrer cette langue au processus d'enseignement. À ce sujet nous pouvons reprendre la réflexion de Geunier : "Si la «valeur » du langage relève de la parole et non de telle ou telle langue particulière, dans quelles limites la traditionnelle question de l'unicité absolue de cette langue au moment de l'acquisition garde-t-elle sa pertinence? (Geunier, 1982 : 83).

Afin de prendre en compte le vécu et l'histoire des apprenants, nous avons mis en pratique une approche sociobiographique par le détour des fables et des contes. Cette démarche tente de les guider à travers une complexité culturelle, symbolique et imaginaire liée à leur situation de mobilité, d'expatriation et aux processus d'hybridation et de métissage associés (Laplantine, 2002 ; Molinié, 2015). À la suite des concepts développés par Molinié, nous avons construit notre programme de formation dans le but de mieux comprendre et d'accompagner la construction d'une identité plurielle et les processus d'acculturationenculturation. Il a été en effet constaté que les apprenants en situation exolingue "découvraient qu'une discontinuité s'instaurait entre leurs pensées et la formulation de celle-ci pour nouer un dialogue avec les autre » (Molinié, 2015 : 143). La formation dans son ensemble et en particulier les séances présentées dans cet article visent à accompagner les migrants dans le dépassement de cette difficulté au moyen d'une stratégie de retraductionreformulation de leurs actes de parole et de leurs récits de vie. Au cours de l'action de formation, l'apprenant peut ancrer ses apprentissages dans son expérience émotionnelle et se réinventer au monde, s'assumer à la fois pluriculturel et intégré. La formation interroge l'historicité des individus. Elle les invite à entrer dans leur propre histoire et à la relater. Elle encourage l'utilisation de la langue première et la pratique progressive de la langue cible à acquérir. Ce processus d'appropriation se fait à travers la déconstruction des schèmes de l'ordre postcolonial du savoir descendant du prescripteur vers le receveur : au lieu d'être pris par la main du colonisateur et prescripteur linguistique, le migrant vient partager d'égal à égal ses connaissances avec son hôte. Il privilégie le chemin inverse, celui du migrant riche de connaissances "offreur» d'une expérience singulière. Son altérité doit se conjuguer et s'accorder avec celle de ceux avec qui il vit. 


\subsection{Le protocole d'observation des séances}

Lors de la première séance observée les apprenants ont eu pour consigne de choisir un animal représentatif de l'image qu'ils se faisaient d'eux-mêmes. Ils devaient ensuite réaliser un collage sur le dessin de cet animal à partir de mots découpés dans des journaux et magazines locaux. Lors de cette séance, nous avons recueilli la fiche pédagogique détaillée du formateur ainsi que des photographies des productions des apprenants (cf. Figures 1 et 2). Lors de la deuxième séance, les apprenants ont été invités à raconter leur arrivée en Guadeloupe. Ils devaient se raconter sous la forme d'un animal. Il fallait expliquer au groupe les difficultés rencontrées à l'arrivée sur le territoire, puis citer les points positifs et améliorations éventuellement survenues et en tirer des idées sur la meilleure manière que chacun avait trouvé de s'intégrer, socialement, linguistiquement, culturellement, affectivement et économiquement. Dans cette séance, nous avons relevé des traces laissées au tableau et enregistré des interactions orales entre le formateur et les apprenants afin de procéder à une démarche herméneutique (Ricœur, 1969). Ainsi, nous avons recueilli quatre types de traces (des productions d'apprenants, une fiche pédagogique, des traces au tableau et des interactions orales en formation) que nous analysons en vue d'identifier des signatures narratives qui dévoilent l'identité située et en devenir des apprenants et qui révèlent des freins et des motivations à leur intégration dans le pays d'accueil. Nous cherchons à identifier dans ces traces des éléments qui relèveraient, d'une part, chez les migrants, de compétences linguistiques selon leurs fonctions langagières, et d'autre part chez le formateur d'une posture de «passeur culturel engagé » (Molinié, 2015).

\section{Données recueillies et analyse}

Dans cette partie, nous présentons quatre types de données recueillies lors de notre action de formation. Il s'agit, pour la première séance, de la fiche pédagogique du formateur et des productions des apprenants, et pour la deuxième séance, des traces au tableau et des interactions orales entre formateur et participants.

\subsection{Analyse de la fiche pédagogique du formateur}

L'analyse de la fiche pédagogique du formateur (cf. Tableau 1) montre la volonté du choix clair d'une posture d'accompagnement sociobiographique. En effet, nous pouvons y repérer un certain nombre d'éléments visant à impliquer les apprenants en les faisant parler d'eux. Par exemple, dans l'activité $\mathrm{n}^{\circ} 1$, la consigne propose d'expliquer le choix de l'animal en faisant " un lien avec son histoire personnelle ou un souvenir marquant». Dans l'activité $\mathrm{n}^{\circ} 2$, on peut également relever dans l'objectif le passage suivant : "délivrer un message sur ce que je suis ». Ainsi, la fiche pédagogique laisse entendre que le formateur souhaite se baser sur le vécu personnel et expérientiel des apprenants en vue de développer des compétences en langue française. On peut également relever une utilisation de certains leviers psychologiques par un appel à la verbalisation émotionnelle. Ceci est identifiable dans les objectifs de l'activité $\mathrm{n}^{\circ} 1$, "parler de soi en maîtrisant ses émotions" et "insister sur la mise en confiance », et aussi dans l'activité $n^{\circ} 3$, «exprimer son ressenti ». Ceci traduit la volonté du formateur de travailler à la réassurance des apprenants et à les aider dans leur développement personnel. La mise en confiance passe par une auto-analyse de leurs sentiments. Le formateur procède en deux temps, il propose d'abord de décrire, puis d'expliquer («argumenter ce choix » dans l'activité ${ }^{\circ} 1$, "explication par le réalisateur de l'œuvre » dans l'activité ${ }^{\circ} 3$ ). Ainsi l'apprenant est invité à adopter une position métalinguistique qui l'emmène à s'interroger sur les formes de son expression. L'appropriation des savoirs et des savoirs faire 
suit la progression suivante : connaissance, compréhension, application, analyse, évaluation (Bloom, (1975 [1956]). À chaque étape le formateur et le groupe des co-apprenants participent au renforcement des connaissances et à leur mise en œuvre. L'évaluation formative se fait avec l'aide des pairs (activité $\mathrm{n}^{\circ} 4$ " élocution, clarté de la présentation»). L'opportunité de pouvoir observer un individu similaire à soi-même exécuter une activité donnée, constitue une source d'information importante influençant la perception d'autoefficacité (Bandura, 2003). Le formateur utilise l'encouragement par les pairs et l'interaction entre les pairs comme un élément essentiel du processus d'apprentissage (Vygotski, 1985).

\begin{tabular}{|c|c|}
\hline \multicolumn{2}{|c|}{ «PARLE MOI DE TOI » CONTE CREOLE / FABLES DE LA FONTAINE } \\
\hline Scénario pédagogique & Matériel \\
\hline $\begin{array}{l}\text { Présentation individuelle } \\
\text { Présentation des participants }\end{array}$ & \\
\hline \multicolumn{2}{|c|}{ ACTIVITÉ No1 : SE PRESENTER PAR LE BIAIS D'UN SUPPORT : Les personnages de La Fontaine } \\
\hline \multicolumn{2}{|l|}{$\begin{array}{l}\text { Objectifs : *Faire un choix réfléchi, argumenter ce choix. } \\
\text { * Parler de soi en maîtrisant ses émotions. } \\
\text { * Parler à haute voix, distinctement pour être entendu. }\end{array}$} \\
\hline L'objectif est de choisir un élément présent parmi les fables de La Fontaine (un animal ou un personnage). & $\begin{array}{l}\text { Affichage avec la liste des animaux et } \\
\text { des éléments } \\
\text { possibles. }\end{array}$ \\
\hline $\begin{array}{l}\text { Préparation de la présentation de l'animal choisi. } \\
\text { Il s'agit ensuite d'expliquer aux autres pourquoi on a choisi cet animal : ses qualités, ses défauts, son lien éventuel } \\
\text { avec son histoire personnelle ou un souvenir marquant } \\
\text { Insister sur la mise en confiance }\end{array}$ & $\begin{array}{l}\text { Feuilles } \\
\text { Stylos }\end{array}$ \\
\hline \multicolumn{2}{|l|}{ Présentation individuelle de l'animal. } \\
\hline \multicolumn{2}{|c|}{ ACTIVITÉ No2) REALISER UNE OEUVRE PLASTIQUE : M'aider d'un support pour dire qui je suis } \\
\hline \multicolumn{2}{|l|}{$\begin{array}{l}\text { Objectifs: *Réaliser une oeuvre artistique pour délivrer un message sur ce quE je suis, la façon dont je me perçois et } \\
\text { ce que je veux en montrer aux autres. }\end{array}$} \\
\hline $\begin{array}{l}\text { Distribution des supports en noir et blanc représentant les animaux choisis par les participants. } \\
\text { Décalque des contours et des traits principaux de l'animal choisi. } \\
\text { Découpage dans les journaux de mots ou d'images à coller sur le support fourni en relation avec ses propres traits de } \\
\text { caractères à soi ou avec la façon dont on se perçoit par des mots en créole. } \\
\text { Ensuite repassage en noir des traits principaux des animaux : reconnaissance. }\end{array}$ & $\begin{array}{l}\text { Supports avec les animaux } \\
\text { Papier calque } \\
\text { Ciseaux } \\
\text { Crayons } \\
\text { Colle } \\
\text { Journaux en français et en créole }\end{array}$ \\
\hline \multicolumn{2}{|c|}{ ACTIVITÉ N³) S'INTERROGER SUR UNE OEUVRE PLASTIQUE : Comment me percois-je et les autres me percoivent-ils ? } \\
\hline \multicolumn{2}{|l|}{ Objectifs : *Décrire une ouvre, exprimer son ressenti. } \\
\hline \multicolumn{2}{|l|}{$\begin{array}{l}\text { Présentation individuelle des productions } \\
\text { Temps } 1 \text { : Réflexions du public, description des œuvres par les autres. } \\
\text { Temps } 2 \text { : Explication par le réalisateur de l'œuvre des mots ou images collés , utilisés. }\end{array}$} \\
\hline \multicolumn{2}{|l|}{ ACTIVITÉ N4) SAVOIR LIRE A HAUTE VOIX } \\
\hline \multicolumn{2}{|l|}{$\begin{aligned} \text { Objectifs : } & \text { *Préparer la lecture d'une ouvre écrite conventionnelle } \\
& * \text { Lire distinctement à haute voix }\end{aligned}$} \\
\hline $\begin{array}{l}\text { Lecture orale de la fable de La Fontaine liée à l'animal choisi. } \\
\text { Présentation de l'ensemble des supports fables. } \\
\text { A) Choisir sa modalité de travail : Travail en groupe avec répartition des phrases ou présentation individuelle. } \\
\text { B) Préparation de la lecture individuelle et ou collective : Lire dans sa tête, répéter les mots inconnus, rechercher leur } \\
\text { signification. }\end{array}$ & Fables de La Fontaine \\
\hline \multicolumn{2}{|l|}{$\begin{array}{l}\text { Lecture individuelle / collective. } \\
\text { Evaluation formative : prononciation, élocution, clarté de la présentation. }\end{array}$} \\
\hline \multicolumn{2}{|l|}{ Préparation des affichages : Afficher la fable et les productions réalisées } \\
\hline \multicolumn{2}{|l|}{ ACTIVITÉ N5) ATELIER D'ECRITURE } \\
\hline Objectifs : *Ecrire en s'aidant d'un support conventionnel & \\
\hline $\begin{array}{l}\text { Adaptation de la fable choisie aux Antilles } \\
\text { Remplacer les animaux / les personnages de la fable précédemment lu par des animaux / personnages des Antilles. } \\
\text { Intégrer des éléments antillais dans la fable : végétation, lieu maritime, ville, ... } \\
\text { Remplacer des mots puis le contexte petit à petit. }\end{array}$ & $\begin{array}{l}\text { Feuilles } \\
\text { Stylos }\end{array}$ \\
\hline Refaire la production plastique avec des mots en français. & \\
\hline
\end{tabular}

Tableau 1 : Fiche pédagogique du formateur de la première séance 


\subsection{Analyse de productions graphiques des apprenants}

Nous présentons ci-dessous deux productions d'apprenants (cf. Figures 1 et 2) réalisées à partir de la consigne suivante : «Vous devez vous présenter par le biais d'un support. Vous choisissez parmi les images d'animaux suivantes, celles que vous voulez en vue de vous présenter. Ensuite vous découpez dans les journaux des mots qui décrivent votre caractère ou la façon dont vous vous percevez. Puis vous collez les mots sur l'animal comme vous le souhaitez et vous allez devoir expliquer aux autres votre réalisation ». La figure 1 représente une fourmi et la figure 2 un âne. Le tableau 1 reprend les animaux et les différents mots choisis par les apprenants qui ont été collés sur l'image et nous proposons une interprétation thématique et conceptuelle des choix effectués.

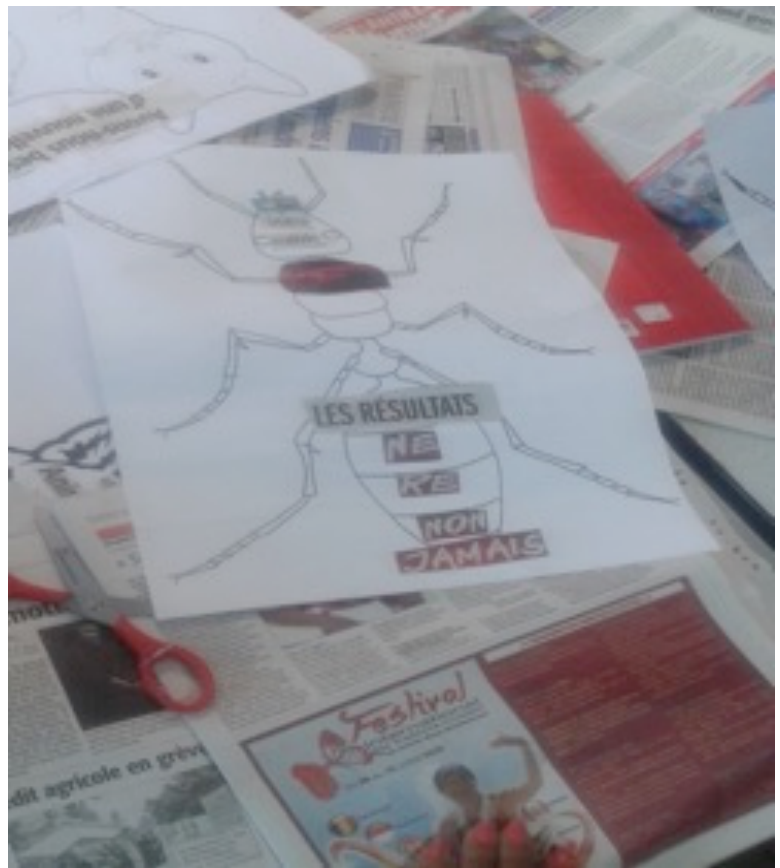

Figure 1 : La fourmi

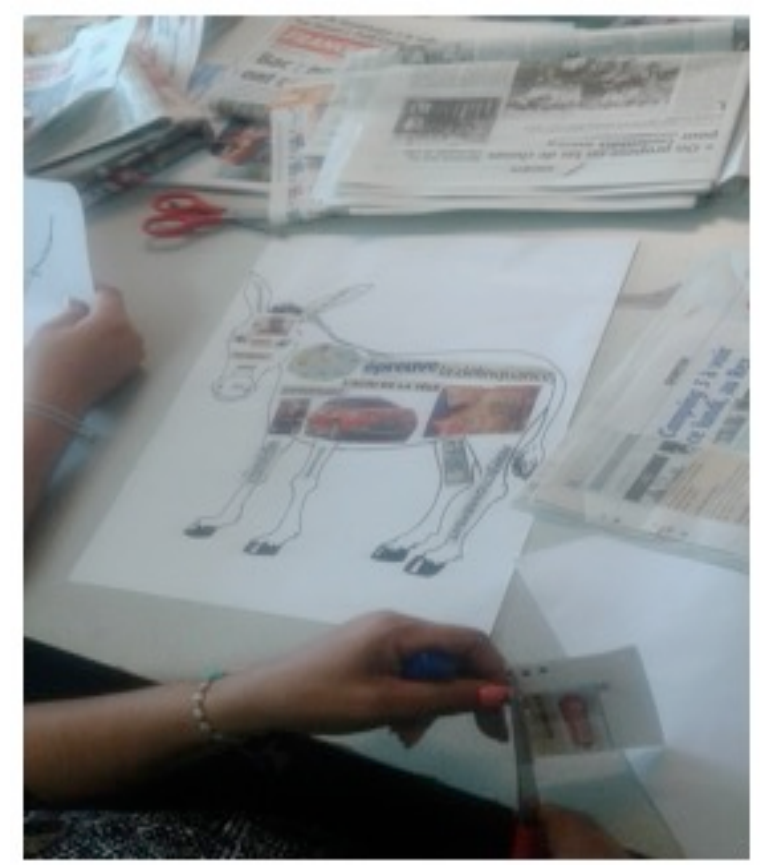

Figure 2 : L’âne

L'analyse herméneutique du tableau fait appel à plusieurs critères d'observation. D'abord celui de la référence à l'animal choisi (colonne A) et le rapport entre le langage et la réalité. Un langage existe soit par un processus de reflet-miroir du monde réel, soit par une dynamique de reconstruction de ce monde réel. Nous trouvons ce critère dans la colonne vocabulaire (colonne B) qui représente ainsi la fonction dénotative consistant à décrire le monde en utilisant le lexique mis à disposition. Nous avons posé une deuxième colonne avec le critère du signe qui se rapporte à ce qui est autre chose, de plus implicite, de sous-entendu (colonne C). Dans la dernière colonne nous avons fait correspondre ce binôme vocabulairesigne au critère de référent qui renvoie à la réalité décrite telle que ressentie et interprétée par le sujet apprenant (colonne D). Il y a donc dans notre démarche interprétative la volonté d'une articulation entre explicite et implicite ; entre objectivité et subjectivité. Ainsi la première colonne du tableau nomme l'animal choisi par l'apprenant. La seconde colonne désigne les mots qu'il a découpés et collés sur l'ombre de l'animal choisi. La troisième colonne propose un classement thématique global de ces mots collés et la dernière colonne propose une interprétation du sens donné à ces mots dans le monde de l'apprenant. 


\begin{tabular}{|c|c|c|c|}
\hline $\begin{array}{l}\text { Animal choisi, } \\
\text { Titre } \\
\text { Colonne A }\end{array}$ & $\begin{array}{c}\text { Vocabulaire } \\
\text { explicite, Signe : } \\
\text { choix de mots pour } \\
\text { décrire son monde. } \\
\text { Colonne } B\end{array}$ & $\begin{array}{c}\text { Sens, Interprétation, } \\
\text { Thématique, Domaine, } \\
\text { Champ culturel : } \\
\text { utilisation possible à } \\
\text { développer en cours. } \\
\text { Colonne } C\end{array}$ & $\begin{array}{c}\text { Analyse conceptuelle, Hypothèses } \\
\text { interprétatives, vocabulaire, } \\
\text { biographie, référent : ancrage } \\
\text { dans la réalité vécue. } \\
\text { Colonne } D\end{array}$ \\
\hline \multirow{2}{*}{ La fourmi } & $\begin{array}{l}\text { Ne renonce jamais } \\
\text { Ligne } 1\end{array}$ & Injonction positive & $\begin{array}{l}\text { Slogan, entrée symbolique, voire } \\
\text { idéologique, correspond à un slogan } \\
\text { publicitaire local, donc appropriation } \\
\text { culturelle même involontaire. }\end{array}$ \\
\hline & $\begin{array}{l}\text { Les résultats } \\
\text { Ligne } 2\end{array}$ & $\begin{array}{l}\text { Norme, objectif, } \\
\text { obligation }\end{array}$ & $\begin{array}{l}\text { Personne jeune et scolarisée, } \\
\text { parallèle effectué entre formation vie } \\
\text { courante. }\end{array}$ \\
\hline \multirow{7}{*}{ L'âne } & $\begin{array}{l}\text { Epreuve } \\
\text { Ligne } 3\end{array}$ & $\begin{array}{l}\text { Difficultés, objectifs, } \\
\text { volonté. }\end{array}$ & $\begin{array}{l}\text { Rapport aux difficultés d'intégration, } \\
\text { de compréhension }\end{array}$ \\
\hline & $\begin{array}{l}\text { Anniversaire } \\
\text { Ligne } 4\end{array}$ & $\begin{array}{l}\text { Moments de vie, repères } \\
\text { temporels, vie de famille. }\end{array}$ & $\begin{array}{l}\text { Recherche de convivialité, de } \\
\text { regroupement social, moments } \\
\text { commémoratifs collectifs. }\end{array}$ \\
\hline & $\begin{array}{l}\text { La délinquance } \\
\text { Ligne } 5\end{array}$ & $\begin{array}{l}\text { Société, actualités, } \\
\text { préoccupations. }\end{array}$ & $\begin{array}{l}\text { Problématique sociale locale, thème } \\
\text { récurrent de conversations, peur } \\
\text { collective, préoccupation partagée. }\end{array}$ \\
\hline & $\begin{array}{l}\text { L'actu de la télé } \\
\text { Ligne } 6\end{array}$ & $\begin{array}{l}\text { Quotidien social, médias, } \\
\text { informations, } \\
\text { occupations ménagères. }\end{array}$ & $\begin{array}{l}\text { Sujets de conversation, socioculturel } \\
\text { rassurant, fonction psychologique } \\
\text { contrephobique. }\end{array}$ \\
\hline & $\begin{array}{l}\text { Bon appétit } \\
\text { Ligne } 7\end{array}$ & $\begin{array}{l}\text { Quotidien partagé, } \\
\text { convivialité, échange, } \\
\text { communication, contact } \\
\text { « universel » }\end{array}$ & $\begin{array}{l}\text { Outil de communication inter } \\
\text { culturel, identification « ethnique » } \\
\text { par la gastronomie. }\end{array}$ \\
\hline & $\begin{array}{l}\text { Perfectionnement } \\
\text { Ligne } 8\end{array}$ & $\begin{array}{l}\text { Objectif, obligation, } \\
\text { injonction, volonté, } \\
\text { recherche de mieux. }\end{array}$ & $\begin{array}{l}\text { Rapport entre les besoins de } \\
\text { performance actionnelle et la } \\
\text { recherche de reconnaissance de la } \\
\text { réussite des tâches communicatives } \\
\text { imposées par le système. }\end{array}$ \\
\hline & $\begin{array}{l}\text { Comédie } \\
\text { Ligne } 9\end{array}$ & $\begin{array}{l}\text { Loisirs, amusement, } \\
\text { développement culturel. }\end{array}$ & $\begin{array}{l}\text { Occupations, stratégie d'évitement } \\
\text { des problèmes d'adaptation. }\end{array}$ \\
\hline
\end{tabular}

Tableau 2 : Analyse de deux productions graphiques des apprenants de la première séance

L'étude du mot présenté à la ligne 1 («ne renonce jamais ») mis en relation avec ceux de la ligne 2, 3 et 8 ( « les résultats, épreuve, perfectionnement») traduit une volonté de répondre à des injonctions. En effet, une dynamique de formation transparait chez l'apprenant à travers ces mots dans le fait de vouloir atteindre des objectifs, de rester motivé, de surmonter des difficultés. Cette volonté semble devoir être assujettie à une obligation de résultats. La notion de difficulté transparait à travers le choix du mot " épreuve» et les objectifs de la mise en formation à travers le mot «perfectionnement». Cet aspect de la motivation des apprenants en formation indique l'importance de l'une des particularités du dispositif présenté : le caractère optionnel de la formation, non obligatoire et basée sur le volontariat. Ce caractère s'oppose à la forme habituelle du dispositif FLI où les apprenants sont forcés par les administrations compétentes (Office français de l'immigration et de l'intégration, désormais OFII ; et la Préfecture de Guadeloupe) de suivre un cursus contraignant sur une durée de plusieurs mois. Cet aspect rend encore plus problématique la dimension postcoloniale de l'organisation des apprentissages du français dans le contexte antillais. En effet, c'est la 
France hexagonale qui fixe les règles et les obligations. Elle peut le faire sans tenir compte des spécificités sociolinguistiques du territoire. Cet aspect coercitif vient renforcer le sentiment de double identité qui est commun aux migrants et aux citoyens français de Guadeloupe. Il est possible que chez ces deux populations qui se fréquentent existe une volonté d'émancipation en réaction à ces obligations venues de Métropole. Dans les deux cas, c'est un désir de liberté qui s'exprime en même temps qu'une préoccupation d'obéissance aux normes, normes métropolitaines pour la Guadeloupe, normes du pays d'accueil pour les migrants. Entre émancipation et obéissance, l'injonction peut être contradictoire et poser un problème de construction identitaire récurrent.

C'est ici que la dynamique de partage d'une situation postcoloniale des apprenants et de ceux qui les accueillent fait sens. Cette spécificité culturelle pourrait être didactisée sous la thématique de la double identité. Elle existe chez le migrant fidèle à sa culture d'origine et désireux de s'intégrer, comme elle existe chez le guadeloupéen né de la colonisation et désirant s'autonomiser. La pédagogie de la variation vient dans cette partie de notre expérience se conjuguer avec la pédagogie des approches sociobiographiques pour permettre de révéler des schémas d'intentions chez l'apprenant en lui donnant des moyens détournés de s'exprimer et de se révéler. "Le biographique agit comme un instrument puissant qui permet une réunification du sujet en lui proposant aussi une expression de ses affects » (Deprez, 1996). Avec l'étude des mots présentés lignes 4, 7 et 9 (anniversaire, bon appétit, comédie), les problématiques individuelles des apprenants s'articulent avec des préoccupations plus collectives qui font sens au niveau de la vie en société. Ceci appelle à une analyse des intentions à plusieurs niveaux d'échelle. La vignette clinique de l'échantillon met en tension la possibilité de relever un défi pédagogique. "Le défi est de faire en sorte que tout ce qui se pratique en dehors et à côté de la langue de scolarisation bénéficie à cette dernière et, inversement, que la manière dont celle-ci est travaillée et développée permette aussi l'ouverture sur la pluralité » (Castellotti, Coste, Duverger, 2008 : 12).

\subsection{Analyses des traces au tableau}

La figure 3 est une photographie des traces laissées au tableau par le formateur. Elle propose le carcan d'une progression afin de guider l'expression des apprenants. Cette progression se fait en six étapes numérotées. La première ligne propose de s'exprimer à la première personne du singulier et d'utiliser le verbe être au présent de l'indicatif. Elle est complétée par une formulation déictique qui utilise voici et le possessif mes à côté du terme d'aventures. L'apprenant est ainsi invité à s'investir dans un récit et à y mettre ses impressions personnelles, donc biographiques. La deuxième ligne montre comment écrire une date et situer une action dans un lieu. La troisième ligne passe par une onomatopée, ouhaou, qui est suivie de deux adjectifs placés en attributs et accordés au singulier et au féminin : émerveillée, impressionnée. Les quatrième et cinquième lignes introduisent les adverbes malheureusement et heureusement, comme déclencheurs de l'évènement narratif. Ils sont liés à une volonté d'exprimer des éléments négatifs puis positifs. L'ordre est important puisqu'il correspond à une volonté de nommer d'abord des difficultés pour décrire les éléments qui ont ensuite permis de les résoudre. Ces deux adverbes sont adossés à des repères correspondant à l'introduction de personnages qui représentent autrui dans le schéma narratif de l'aventure. La dernière ligne propose l'item moralité et donne l'exemple d'une petite phrase courte sous forme d'assertion : à deux c'est mieux. La variété des possibilités d'un travail grammatical est importante à l'intérieur du carcan proposé sur ce tableau. À partir du récit construit par les apprenants, le formateur favorise le transfert de notions grammaticales normées. Il travaille sur les accords en genre et en nombre (ligne 3), sur l'accent à mettre sur la lettre $a$ et sur les 
différences entre majuscules et minuscules (lignes 2 et 6), sur l'orthographe du nom d'une ville (New-York, ligne 2), et peut ainsi varier à volonté en proposant d'écrire le nom de n'importe quelle ville de France ou du pays d'origine des apprenants. Il peut travailler la prononciation et le nombre de syllabes des mots, cinq pour malheureusement, quatre pour moralité. Il peut aborder la transformation des verbes en participes comme avec trouver, trouvé (ligne 4)... Le fait de numéroter la présentation au tableau permet également de travailler la compréhension de la sémiographie et l'ordonnancement d'une information.

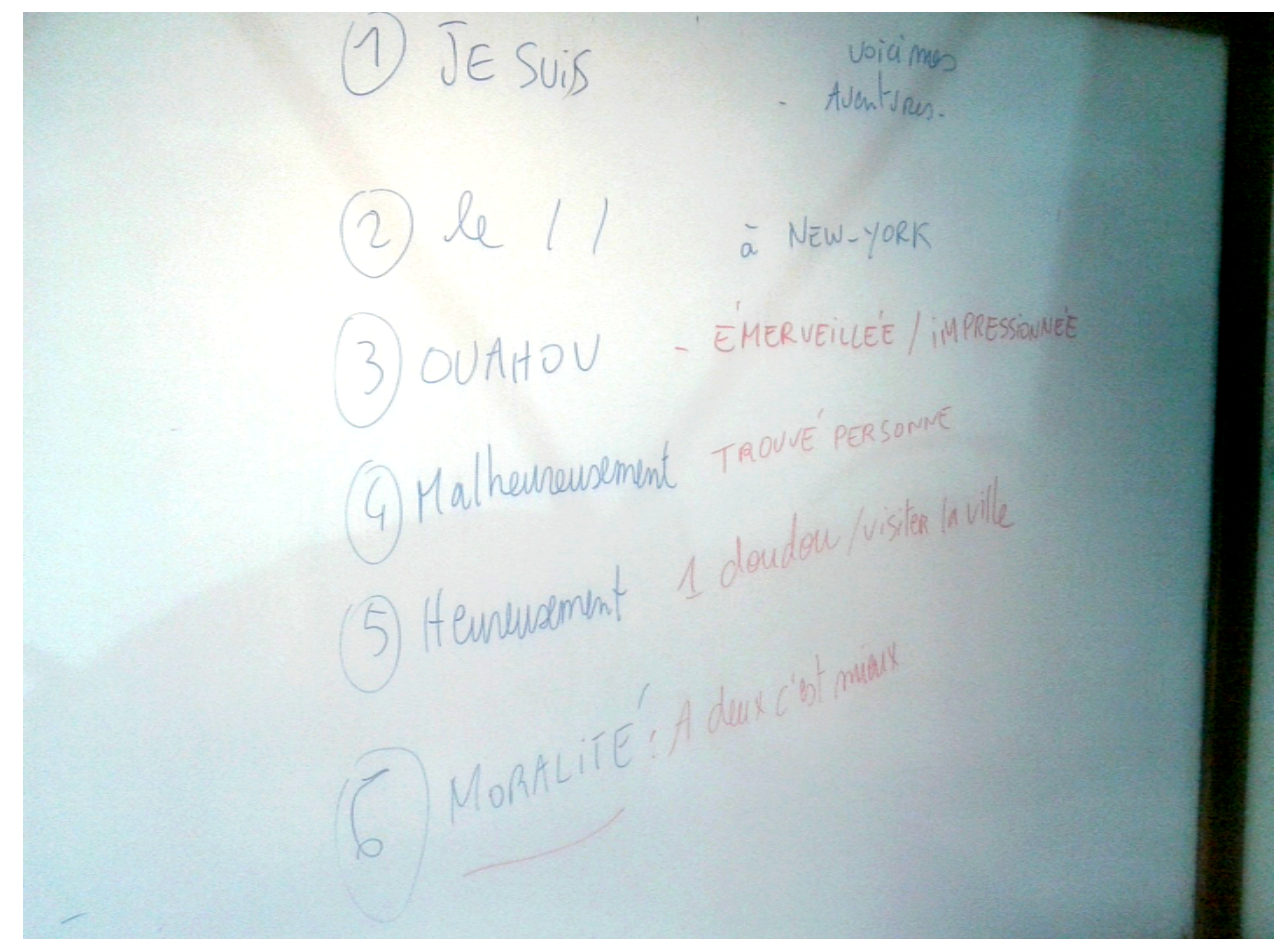

Figure 3 : Traces du tableau de la deuxième séance

\subsection{Analyse de traces d'interactions orales}

Voici un extrait de la retranscription des échanges verbaux entre le formateur et les apprenants lors de la deuxième séance que nous commenterons par la suite :

1. Le formateur: «Voilà un petit peu d'écriture créative, on est le 27 septembre 2016. Comme la formation s'appelle « parle-moi de toi », comment on dit en espagnol ?»

2. Un apprenant : « Habla me de ti »

3. Le formateur : " Habla me de ti. Parle-moi de toi. Il faut quand même que vous arriviez à parler de vous. Et comme on est dans les fables de La Fontaine, on va le faire à la façon des fables de La Fontaine et on va le faire en français aujourd'hui, hein, on oublie un petit peu le créole, on laisse un peu l'espagnol de côté sauf que moi je fais des traductions, des explications. Vous avez le droit de parler en espagnol mais on essaye aujourd'hui d'aller vers le français. Et on a choisi un animal, la consigne : «moi je suis un renard, ou moi je suis une fourmi, moi je suis... (Le formateur se dirige vers chaque apprenant et le désigne du doigt pour l'inviter à se choisir un animal).

4. Un apprenant : «Panthère »

5. Le formateur : «Fais la phrase en entier»

6. L'apprenant : «Moi, je suis un panthère »

7. Le formateur : «Une panthère »

8. L'apprenant : « Une panthère »

9. Le formateur : «Il y a le masculin et le féminin »

10. Un autre apprenant: «Moi je suis un lapin»

11. Le formateur : « Oui »

12. Un autre apprenant : « Moi je suis un chatte» 
13. Encore un autre apprenant : « Moi je suis oun'rate»

14. Le formateur : « Alooooors, vous allez raconter : « Voici mes aventures », au pluriel avec un S. Le, on met la date, caché (e) dans la valise pour la fourmi, dans le moteur pour le lion ou le tigre, j'ai un tigre dans le moteur... »

15. Un apprenant : «Les ailes»

16. Le formateur : « Dans les ailes si tu veux. Je suis arrivée, ée en Guadeloupe. Pourquoi on a mis un E ? Parce que c'est la marque du...? ?

17. Un apprenant : « Du pluriel»

18. Le formateur : «Non, du féminin, pour les filles, d'accord ? Ça change pas la prononciation à l'oral, mais à l'écrit ça change quelque chose. Ça marche?»

Dans cet extrait, le formateur commence par accorder une place dans l'échange à la langue première des apprenants en demandant la traduction du titre de la formation en espagnol (ligne 1). Cela témoigne d'une volonté d'intégrer la langue des migrants dans l'échange et donc d'une pédagogie de la variation qui consiste à partir des langues en présence en vue d'acquérir de nouvelles connaissances. Ensuite, le formateur demande aux apprenants de parler d'eux mêmes en choisissant un animal (ligne 3). Cette démarche, que l'on peut qualifier de sociobiographique, utilise une dimension imaginaire, culturelle et symbolique pour amener les apprenants à s'exprimer. Ce détour par un avatar est un outil pédagogique pour favoriser la mise en mot de soi. Après avoir accordé une place à la langue des apprenants et à leur choix d'animal, le formateur passe à des aspects plus linguistique et formel. Il commence par demander la formulation d'une phrase simple (ligne 5), puis il aborde la question du masculin et du féminin (ligne 9), ainsi que la notion de pluriel (ligne 14). Ainsi dans cet extrait, on peut relever que l'utilisation d'une pédagogie de la variation et d'une approche sociobiographique est susceptible d'introduire l'apprentissage de certaines règles de la langue en FLI.

\section{Discussion}

L'analyse du tableau 1 montre que la séance vise un objectif d'appropriation par les participants mis en position d'apprenants par une convocation de leur accord explicite à entrecroiser les résultats de leurs productions graphiques, orales, et finalement écrite. Ces échanges sont effectivement « régulés » par le formateur. Le travail d'écriture final va donner lieu à une transposition vers des savoirs «relativement normés». Le choix de « relativement» s'explique par une volonté pédagogique de faire progresser «l'apprenti francophone » sur la base de ce qu'il connait déjà, mais surtout sur celle de «ce qu'il est déjà », de « qui il est déjà ». Le formateur dirige les apprenants vers l'acquisition d'une norme concertée correspondant à une temporalité progressive de leur histoire de vie vers leur histoire «à vivre ». Il propose l'utilisation de concepts pour penser français à partir de la «pensée créole » (Hoareau, 2010). Il s'agit de comprendre comment pense l'apprenant pour parvenir à lui faire saisir la façon dont un français pense. Ceci pose la question d'identifier si c'est possible comment pense un français des Caraïbes. À ce titre il faut d'abord considérer le "penser » caribéen, métissé, antillais, à la fois français et créole. L'utilisation de la langue créole en Guadeloupe aux côtés de la langue française suggère en effet un mode de pensée particulier, un « penser créole ». À partir du programme de la fiche pédagogique du formateur de la première séance observée, nous avons cherché à identifier les paradigmes sur lesquels s'appuie le formateur pour didactiser les évocations des apprenants et quels axes de transposition didactique il a mis en œuvre pour rendre ces évocations sources d'apprentissage.

Nous constatons que le formateur développe une approche de l'altérité et du diversitaire en commençant par proposer aux apprenants de faire un choix personnel. Celui d'un animal des fables qu'ils pensent pouvoir les représenter. Ce choix se fait en face des autres apprenants, 
selon une approche socioconstructiviste. Ainsi il pluralise et il « hétérogénéise » le panel des possibles à exploiter en cours. Ensuite, il met l'accent sur l'aspect autobiographique du travail proposé en demandant de relier l'animal à un épisode de la vie passée ou présente du participant. Ainsi il met en exergue le «pourquoi, pour/quoi» apprendre afin de "réintroduire un questionnement fondamental (...) et de distinguer ce qui relèverait, d'une part, d'un "apprendre la langue" et ce qui, d'autre part, serait sous-tendu par un réel projet d'appropriation, qu'il soit ou non explicite» (Castellotti, 2015: 268). Puis il se fait facilitateur d'un processus d'inter-compréhension en invitant à l'explicitation de ce choix, ce qui permet donc d'exprimer et de clarifier le projet de vie de chacun, mais aussi de mesurer la compréhension de l'exercice. Le travail sur la compréhension est différent de celui sur l'évaluation. Il ne s'agit plus de certifier l'acquisition d'habiletés de langage liées à des soidisant besoins de communication ou réussites à une tâche actionnelle imposée ; mais plutôt de travailler sur "l'in-compréhension» et de l'utiliser comme sources d'apprentissages. En faisant travailler l'incertitude par une autorisation d'énoncés formant des empans d'inexactitude, le formateur ouvre une brèche dans le processus d'insécurité (langagière) et l'utilise comme "motrice de l'appropriation» (Castellotti, 2015 : 277). Enfin, il favorise la confrontation du jugement (bienveillant) des productions réalisées entre les apprenants. En utilisant cette didactique de l'altérité par l'altérité, il autorise l'utilisation du levier de la cotraduction pour «regarder les langues» (Diagne, 2013). Par son accompagnement sociobiographique, le formateur contribue à la construction d'une identité pluriculturelle de l'apprenant. L'approche par utilisation du principe de la zone proximale de développement qui ressort de l'analyse de cette fiche pédagogique équivaut à une démarche d'accompagnement sociobiographique par la médiation culturelle car « seule la présence d'un médiateur permet une progression dans la ZPD» (Berdal-Masuy, Briet et Pairon, 2004).

L'analyse du tableau 2 montre une possibilité de transposition didactique qui peut prendre pour point de départ le « déjà-là » langagier des apprenants (Perregaux, 2002, 2004). Cette transposition doit tendre vers une clarification du signifié. La progression étant ensuite de proposer une nomination la plus proche possible du français normé à acquérir, en proposant un classement des lexèmes et archilexèmes: une table est un meuble, la peur est un sentiment... Contrairement à la méthode d'enseignement par l'approche actionnelle où le signifié est donné de prime abord sous forme d'objectifs à atteindre pour réussir à provoquer la compréhension chez l'interlocuteur, il est possible de produire cette réussite en mesurant une réaction en retour à l'énoncé produit initialement. Puisque nous avons vu qu'il existe plusieurs signes possibles pour un même référent, nous supposons ces signes peuvent varier en fonction de nombreux critères (niveau d'études, origine sociale, difficultés cognitives, niveau d'attention, proximité lexicale entre la langue source et la langue cible, problème de faux-amis...). La remédiation des séances de FLI qui tenteront d'appliquer le principe herméneutique présenté dans ce tableau devra tenir compte de cette variation des items évoqués et réguler l'acquisition de leurs différentes significations. Dans cette optique, il n'est alors pas interdit au formateur d'utiliser les langues source des apprenants (créole, anglais ou espagnol) pour les inviter à expliciter leur choix de mots tel qu'il est présenté dans le tableau 2. Le formateur peut poser la question : pourquoi as-tu choisi cet animal ? Pourquoi as-tu collé ce mot ? Dis le moi dans ta langue. Et ainsi faire appel à la personnalité des apprenants qui peuvent produire une infinité de phrases à partir d'un nombre fini et limité de règles (Chomsky, 1971). Il s'agit bien d'une démarche de régulation et d'accompagnement du " déjà-là » langagier et culturel. Il s'agit ensuite de les amener à prendre conscience de leurs habitus ancrés tels des empreintes sociales laissées par les divers systèmes d'interdépendance au sein desquels ils agissent (Elias, 1991). Il s'agit bien d'accompagnement et d'émancipation. 
L'analyse de la figure 3 des traces au tableau montre une dynamique pédagogique particulière. La dynamique de cette séance relève d'un travail de transposition basé sur la capacité analogique de l'apprenant. Ici, nous passons de l'analogie contextuelle travaillée plus haut à travers les productions graphiques à une analogie inclusive qui inclut l'apprenant à l'apprentissage au moyen de la verbalisation guidée d'un récit, mettant en œuvre une transposition didactique régulée. Cette régulation se fait à partir du carcan posé au tableau par le formateur. Notre analyse nous amène à décrypter à l'intérieur des traces laissées au tableau par le formateur les éléments qui peuvent être apportés à la compréhension des apprenants lors de l'accompagnement de leurs récits guidés. Outre l'aspect grammatical, ces éléments correspondent à l'acquisition d'outils linguistiques favorisant la mise en œuvre des fonctions du langage. Afin que le message produit par les apprenants s'exprime à l'intérieur d'un référent, le contexte du récit des aventures de la personne est proposé. Le cadre géographique oriente le discours. Il correspond à la fonction référentielle du langage en renvoyant le message vers une correspondance au monde extérieur. D'abord l'exercice a été réalisé avec la consigne de raconter son arrivée en Guadeloupe puis il a été proposé d'imaginer un voyage à New-York selon les mêmes modalités. Ainsi l'apprenant peut utiliser les schémas langagiers articulatoires vus et les transposer dans un autre contexte : il utilise la langue pour dire sa pensée et traduire la volonté de ses actes, car « dire c'est faire » (Austin, 2005 [1962]). Le message requiert également un code commun qui est matérialisé par la hiérarchisation des énoncés par ordre chronologique ainsi que par la norme d'écriture correcte d'une date et d'un lieu topologique et l'utilisation du «à » avec accent. Ceci correspond à la fonction métalinguistique où le code devient lui-même l'objet du message. Tout au long de l'exercice sont maintenues les fonctions phatique (pour la mise en place et le maintient de la communication) et expressive (pour l'expression des sentiments du locuteur) par une invitation à énoncer verbalement à l'oral le récit en même temps que sa construction se fait à l'écrit au tableau. Ceci permet de maintenir un contact entre les participants, une connexion physique et psychologique interactive. Ainsi, les fonctions du langage étudiées «ne s'excluent pas les unes les autres, mais (...) souvent elles se superposent » (Jacobson, 1960).

L'introduction progressive de normes grammaticales se fait à l'arrivée des adverbes « malheureusement » et " heureusement » qui participent également à la fonction poétique du langage en étant placés au début de la phrase. Ainsi, le caractère « euphonique » est maintenu dans une esthétique minimale et l'ordre des mots dans la phrase est étudié de manière transversale. Avec l'onomatopée « ouhaou » le ton et la hauteur de voix sont soulignés dans l'expression de l'enthousiasme qui peut être comparé culturellement: comment exprimezvous votre joie, votre surprise... dans votre langue maternelle ? Avec quelles expressions, quelles onomatopées... ? Le rapport avec le thème général de la formation est assuré par la recherche d'une moralité finale comme dans les fables de La Fontaine où peut être travaillé le sens explicite et le sens implicite d'un énoncé à caractère universel. Cette phase permet de résumer le travail effectué et d'en tirer des leçons de morale qui articulent chacune des étapes racontées. Dans notre exemple, la moralité « à deux c'est mieux » fait référence au fait que voyager seul est source de difficultés de communication là où l'aide d'un médiateur linguistique peut s'avérer utile voire indispensable. L'apprentissage des schémas langagier se fait de manière progressive en partant de la capacité des apprenants à entrer dans l'imaginaire et le symbolique.

L'analyse des traces d'interactions orales de la deuxième séance montre que le formateur a su, d'un côté, offrir la possibilité à chacun d'exprimer sa personnalité à l'intérieur du groupe, et d'un autre côté, de travailler sur la langue comme un système de codes à faire acquérir. " Il 
faut de l'autre, donc à la fois de l'écart et de l'entre, pour promouvoir du commun » (Jullien, 2011: 72). Cette démarche propose de travailler sur la sensibilité herméneutique de l'apprenant pour exprimer son projet et à travers lui son identité et son rapport au monde. Il relève dans l'évocation de sa propre histoire (raconter son arrivée en Guadeloupe, décrire son propre caractère et ses projets à travers le choix d'animaux significatifs d'aspects particuliers) des significations symboliques et le travail du formateur est de l'aider à lui donner du sens et des outils linguistiques servant à décoder-exprimer ce sens. C'est cette posture qui a été au cœur des enjeux présentés dans la présente séance. Il s'agit d'amener à la compréhension de «l'expérience du monde social » (Gadamer, 1996 : 20).

L'approche régulée d'accompagnement sociobiographique présenté dans notre expérience peut être comprise comme étant un adjuvent méthodologique du FLI. L'analyse de nos résultats montre que les exercices proposés aux apprenants ont facilité leur progression et leur repérage au sein de leur zone proximale d'acculturation. Cet accompagnement se situe en Guadeloupe dans la lignée d'études précédentes réalisées dans le champ de la psychologie interculturelle qui nous renseignent sur cette forme de médiation propre à l'acculturationintégration: "cette médiation opérerait dans ce que l'on pourrait appeler une zone de proche développement acculturatif ou "zone proximale d'acculturation » (ZPA) agissant par le biais d'activités favorables à l'installation de nouveaux schèmes de pensée et de se penser dans un contexte social et culturel dominant. Cette logique valorise la fonction de "médiateur d'acculturation". Ainsi, le médiateur accompagnerait le novice dans une activité nouvelle et accessible afin que ce dernier puisse en intérioriser les aspects -le programme, le script... cognitif et méta-cognitif afin de devenir autonome dans cette activité» (Mokounkolo et Pasquier, 2008: 59). L'objectif du formateur est dans le cadre d'un accompagnement sociobiographique d'amener ce processus d'acculturation vers un transformable des habitus qui aille dans le sens d'une intégration, là où « On parle d'intégration quand ils (les migrants) maintiennent des éléments de leur culture d'origine tout en empruntant ceux du groupe hôte. (Mokounkolo et Pasquier, 2008 : 58). C'est la volonté affichée dans la fiche du formateur que nous avons analysée et qui correspond à une contextualisation régionale des apprentissages tout comme les conclusions que nous avons tirées de l'analyse des interactions orales de la séance suivante. La volonté de faciliter l'intégration des migrants par des approches plurielles et par leur accompagnement à l'évocation biographique en contexte est le leitmotiv de la formation expérimentale présentée. Nous constatons que les objectifs ont été atteints au moins en ce qui concerne la motivation et la réassurance des apprenants qui ont tous demandé une suite de parcours à la fin de cette formation. Il semblerait que l'accompagnement sociobiographique en contexte dans l'enseignement du français langue d'intégration en Guadeloupe soit un facilitateur d'appropriation de connaissances linguistiques, culturelles et un régulateur de la motivation psychologique et affective des apprenants migrants. Notre démarche propose de partir du " déjà-là » langagier, affectif et culturel des apprenants pour les mener, progressivement vers l'appropriation des normes orthoépiques de la langue cible. La démarche rappelle le besoin de l'apprenant de s'approprier son apprentissage et revisite la dimension de transposition didactique en contexte. Le formateur axe ses objectifs sur une pédagogie de l'altérité et cherche à ancrer les apprentissages dans le projet de vie des apprenants. Il utilise l'intercompréhension entre participants. Faire acquérir aux migrants conjointement les deux aspects de la culture guadeloupéenne, française et caribéenne, revient à inscrire notre action dans le cadre d'une action pédagogique relevant des approches plurielles où les langues sont en contact interactif et où convergent sous ce terme générique les approches de la didactique interculturelle, didactique des langues intégrées et l'éveil aux langues (Candelier, 2008). Ainsi l'accompagnement sociobiographique des migrants s'inscrit dans un cadre conceptuel élargi. 
L'analyse des séances observées et des traces laissées à leur suite montre qu'il est possible d'identifier des compétences linguistiques intégrées à partir du récit de vie des apprenants. Une posture bienveillante d'accompagnement de ces récits est facilitatrice de la mise au jour de ces compétences. Elles peuvent être réinvesties dans un apprentissage du français. Cependant, il est nécessaire de considérer le contexte créolophone et biculturel de la Guadeloupe. La démarche est politique et sera différente selon que l'intention est d'intégrer les migrants à tout le territoire de la République, auquel cas il est utile d'utiliser des références culturelles nationales et littéraires historiquement liées à la culture francophone, ou selon que l'on considère que les migrants sont déjà en partie intégrés de fait au sein de la zone Caraïbe. La contextualisation des apprentissages est laissée au bon vouloir des formateurs par le Cadre Européen des Référence pour les Langues qui «n'a pas vocation de promouvoir une méthode d'enseignement particulière mais bien de présenter des choix» (CECRL, 2001: 110, dans Riquois, 2014 : 94). Le formateur se heurte à une position paradoxale défendue par ce même cadre qui "impose des notions fixes uniformes et qui tend à gommer les particularités locales au bénéfice d'un discours unificateur »(CECRL, 2001: 110, dans Riquois, 2014 : 94-95). Dans l'optique de contourner cette difficulté, la régulation des enseignements proposée dans la formation analysée se sert d'excuse : l'artefact d'un avatar (ici un animal choisi dans des fables) permet de décentrer l'apprenant de ses affects tout en le reliant inconsciemment à son vécu pré et post intégratif dans le pays d'accueil. Il adopte sans s'en rendre compte une position méta-existentielle et procède à une conscientisation de sa personnalité migrante et en devenir. Le travail du formateur est de relier cette conscientisation à des outils d'expression linguistiques afin que s'opère un glissement sémantique vers la langue cible. L'avantage est que ceci se fait sur la base de réels besoins, parfois non explicités auparavant, et non sur un présupposé de besoins de réalisation de tâches actionnelles mesurées par des indicateurs de performance décontextualisés.

Dans ce cas, et à la suite des évolutions qui ont historiquement eu lieu dans le domaine de la didactique des langues pour migrants, passée des méthodes structuro-globale audiovisuelle dans les années 1970, à l'approche communicative et après les années 2000 à l'approche actionnelle, nous dessinons la possibilité d'une nouvelle rupture épistémologique vers une approche "régulatrice » complémentaire des précédentes, qui épouserait les théories d'une approche (plus) diversitaire (Castellotti, 2015). La finalité associée aux tâches à réussir y est directement liée à une utilité personnelle pour les participants. Elle advient en contexte « in vivo ». Les participants sont concernés. Il faut qu'ils puissent faire le lien entre leurs apprentissages et l'utilisation qu'ils peuvent en faire une fois sortis du cours. Or, si l'on admet qu'en Guadeloupe la langue de communication sociale n'est pas uniquement le français normé il faut également accepter que cette seule norme ne suffit pas à assurer ce lien de double contextualisation nécessaire : contextualisation à l'environnement social extérieur, et contextualisation à un lien logique rattachant l'apprenant à son histoire, à ses besoins réels, et à ses projets. Une pédagogie de la variation et de la diversité des langues en présence permettrait une réelle intégration des populations migrantes vers une réussite de tâches pragmatiques. En cela, la méthodologie de la régulation par l'accompagnement sociobiographique est une posture pédagogique contextualisée. Cette approche emploie des outils a-scolaires afin d'optimiser les possibilités d'appropriation affectives et cognitives en utilisant des vecteurs de médiation culturelle et artistiques : récits, contes, jeux de théâtre, collages papiers, photographie, poésie... La régulation est proche de la notion de médiation. Cette médiation se fait par le moyen d'allers / retours entre la langue source et la langue cible, entre le "déjà-là » et le " à comprendre ", afin de réguler la progression vers un consensus lucide sur une intégration sans désintégration. La posture du formateur change et passe de 
formateur prescripteur en FLI à celle de formateur accompagnateur-passeur culturel. En d'autres termes, la culture cible n'est pas donnée à comprendre à partir d'un " mode d'emploi » qui serait uniquement présenté au moyen de normes grammaticales à intégrer. Il va falloir faire un travail de décodage. Malgré tout, c'est de cette confrontation à autrui et entre pairs que pourra naitre la connaissance, et plus particulièrement la compréhension, puis une certaine connaissance et pratique de la langue d'intégration.

\section{Conclusion}

Dans notre expérimentation, la conjugaison des altérités crée une catalyse de l'unicité : le commun est avant tout la superposition des différences de chacun et leur articulation opératoire. Le besoin premier des apprenants est celui de la " compréhension / assimilation » qui mènera ensuite, et seulement dans certains cas, à une «compréhension / utilisation / intégration / régulation » des apprentissages et des pratiques. Il est nécessaire dans cette optique d'adopter une posture éthique différente qui puisse d'abord accepter d'inviter à " fortifier l'aptitude à interroger et (...) lier le savoir au doute, développer l'aptitude à intégrer le savoir particulier non seulement dans un contexte global, mais aussi dans sa propre vie » (Morin, 1999). Il est ainsi recommandé d'accepter de faire dialoguer les aptitudes du « déjàlà » langagier premier et le bagage culturel et affectif des apprenants avec la norme linguistique du pays d'accueil, afin qu'elle s'y ajoute sans renier ce déjà-là. Cette opération aura lieu au travers d'un champ de confrontations et de conflits, propre à l'enjeu de toute rencontre altéritaire (Robillard, 2011).

Nous tentons de montrer la possibilité d'opérer une adaptation didactique du concept de zone proximale de développement cognitif vers celui de zone de développement d'acculturation et d'intégration. Dans cette approche des apprentissages, l'apprenant remet en jeu ses acquis affectifs et culturels à travers le récit de son parcours sociobiographique et tente de trouver les outils linguistiques adaptés au pays d'accueil pour exprimer à la fois son histoire et son projet de vie. Le formateur met en relation les participants et dégage un corpus d'évocations pour prendre ancrage vers l'appropriation des schèmes langagiers vers une intégration négociée. Il développe les compétences d'une intercompréhension sociale nécessaire au vivre-ensemble au sein du pays d'accueil. Cette opération pédagogique doit se faire sur la base d'une mise en valeur de la langue et de la culture d'origine des apprenants. Le but est de se concentrer sur le partage plutôt que sur une assimilation réductrice, encore héritière d'un système de domination de l'immigré par son hôte dominant. En contexte multilingue caribéen, opter pour le monolinguisme peut être un frein à une intégration efficace. Il apparait préférable de parvenir ensemble à un " tout-monde » où les cultures sont conjuguées plutôt qu'absorbées : "J'appelle Tout-monde notre univers tel qu'il change et perdure en échangeant et, en même temps, la "vision" que nous en avons. " (Glissant, 1995). La didactique des langues accompagne alors les transformations de la société au lieu de tenter vainement de les réifier.

\section{Références bibliographiques}

Anciaux, F. (2013). Alternances et mélanges codiques dans les interactions didactiques aux Antilles et en Guyane françaises. Dossier d'habilitation à diriger des recherches, Université des Antilles et de la Guyane.

Austin, J. L. (2005 [1962]). Quand dire, c'est faire. Paris : Seuil.

Bandura, A. (2003). Auto-efficacité, le sentiment d'efficacité personnelle. Paris : De Boeck.

Beaubrun, F. (2016). L'enseignement du Français Langue d'Intégration aux adultes migrants haïtiens en Guadeloupe : de l'insécurité linguistique héritée du scolaire à des modalités 
de remédiation pédagogiques. Colloque Pathologies du langage et rendement scolaire, janvier 2016, Université d'Etat d'Haïti.

Beaubrun, F. (2014). Réflexions sur l'éducation. L'Andragos, Mérignac, Copy-Média.

Berdal-Masuy, F., Briet, G. et Pairon, J. (2004). Apprendre seul, à son rythme et encadré. Études de linguistique appliquée, 134, 173-190.

Bloom, B. (1975 [1956]). Taxonomie des objectifs pédagogiques. Québec: Presses de l'Université du Québec.

Besse, H. et Porquier, R. (1991). Grammaire et didactique des langues, Paris, Hatier / CREDIF, collection LAL.

Candelier, M. (2008), Approches plurielles, didactiques du plurilinguisme : le même et l'autre, Les Cahiers de l'ACEDLE, 5. Actes du colloque ACEDLE.

Castellotti, V. dans Huver et Bel (dir.), (2015). Prendre la diversité au sérieux en didactique / didactologie des langues. Altériser, instabiliser: quels enjeux pour la recherche et l'intervention? Paris, L'Harmattan.

Castellotti, V., Coste, D. et Duverger, J. (dir.) (2008). Propositions pour une éducation au plurilinguisme en contexte scolaire. Paris : Adeb/Dynaviv.

Chevallard, Y. (1986). La transposition didactique: du savoir savant au savoir enseigné, compte rendu. Revue Française de Pédagogie, 76(1), 89-91.

Chevrier, M. (2013). La langue interrompue, revue Arguments.

Chomsky, N. (1971). Aspects de la théorie syntaxique, Paris, Seuil, coll. Ordre Philosophique.

Conseil de l'Europe, (2001). Cadre européen commun de référence pour les langues. Apprendre, enseigner, évaluer, Paris, Didier.

DAIC, Direction ministérielle de l'accueil, l'intégration et de la citoyenneté, (2011). Vicher, A., et Adami, H., Référentiel FLI.

Deprez, C. (1996). Parler de soi, parler de son bilinguisme, Paris : Encrages, p. 155-180.

Elias, N. (1991). La société des individus, Fayard.

Fanon, F. (1961). Les damnés de la Terre, Paris, Librairie François Maspero.

Fergusson, C. (1959). Diglossia, revue World, n¹5.

Frege, G. (1892). Original: Über Sinn und Bedeutung, in Zeitschrift für Philosophie und philosophische Kritik C: 25-50; In English: On Sense and Reference, alternatively translated (in later edition) as On Sense and Meaning.

Gadamer, H. G. (1996). Vérité et méthode. Paris : Seuil.

Georger, F. (2006), Le réunionnais au secours du créole et du français à l'école, dans Etudes Créoles vol. XXVIII n², PP. 119-146.

Geunier, N. (1982), Langue française, langue maternelle et communauté linguistique, Paris, Larousse, $\mathrm{n}^{\circ} 54$, pp.7-28.

Glissant, E. (1995). Tout-monde, Paris, Gallimard.

Grice, H. P. (1981). Presupposition and Conversational Implicature, in P. Cole (ed.), Radical Pragmatics, New York, Academic Press, (pp. 183-198).

Haugen, E. (1962). Schizoglossia and the linguistic norm., Languages and linguistics. $\mathrm{n}^{\circ} 15$, pp 63-69.

Hjelmslev, L. (1975). Résumé of a Theory of Language. Travaux du Cercle linguistique de Copenhague, vol. XVI. Copenhague : Nordisk Sprog- og Kulturforlag.

Hoareau, A. E. (2010). Concepts pour penser créole, éd. Zarkansiel, essais.

Jacobson, R. (1960). Closing statements: Linguistics and Poetics, Style in langage, T.A. Sebeok, New-York, Pour la traduction de Nicolas Ruwet (1963) : «Linguistique et poétique », Essais de linguistique générale, Paris, Éditions de Minuit.

Jeannot-Fourcaud, B. (2016). Insécurité linguistique en contexte guadeloupéen et perspectives didactiques, dans Les cahiers créoles du patrimoine de la Caraïbe, $\mathrm{n}^{\circ} 6$, Canopée éditions. 
Jullien, F. (2011). L'écart et l'entre. Leçon inaugurale de la chaire sur l'altérité, Paris, Galilée.

Laplantine, F. (2002), L'Anthropologie genre métis. Dans Ghasarian, C. (dir), De l'ethnographie à l'anthropologie réflexive, nouveaux terrains, nouvelles pratiques, nouveaux enjeux, Paris, Armand Colin, pp. 143-148.

Ledegen, G. (2002). Les "parlers jeunes » à la Réunion : " $i$ totoch sérieux », Ville-EcoleIntégration-Enjeux, $\mathrm{n}^{\circ} 130$, pp. 133-149. Laboratoire de recherches sur les langues, les textes et les communications dans les espaces créolophones et francophones (LCF-FRE 2564 du CNRS). Université de la Réunion.

Meirieu, P. (2014). Préface. Dans Boudet, M. et Saint-Luc, F. (dir.), Le système éducatif à l'heure de la société de la connaissance. Toulouse : Presses Universitaires du Mirail.

Molinié, M. (dir.) (2006). Biographie langagière et apprentissage plurilingue. Recherches et Applications, 39,

Molinié, M. (2015). Recherche biographique en contexte plurilingue, cartographie d'un parcours de didacticienne. Paris : Riveneuve Editions.

Mokounkolo, R. et Pasquier, D. (2008). Stratégies d'acculturation : causes ou effets des caractéristiques psychosociales? L'exemple des migrants d'origine algérienne. Les Cahiers Internationaux de Psychologie Sociale, 79, 57-67.

Morin, E. (1999). Relier les connaissances, le défi du XXIème siècle. Paris : Seuil.

Perregeaux, C. (2002). (Auto) biographies langagières en formation et à l'école : pour une autre compréhension du rapport aux langues. Bulletin Vals-Asla, 76, 81-94.

Perregeaux, C. (2004). Prendre appui sur la diversité linguistique et culturelle pour développer aussi la langue commune. Repères, 29, 147-166.

Porquier, R. et Py, B. (2004). Apprentissage d'une langue étrangère : contextes et discours, Paris : Didier.

Prudent, L. F. (2005). Interlecte et pédagogie de la variation en pays créoles. Dans L. F. Prudent, F. Tupin, F. et S. Wharton (dir.), Du plurilinguisme à l'école. Vers une gestion coordonnée des langues en contextes éducatifs sensibles. Genève : Peter Lang.

Prudent, L. F., (1981). Diglossie et interlecte. Langages, 61, 13-38.

Romani, J.-P. (1994). Interlecte martiniquais et pédagogie. Études Créoles, 17(1), 84-105.

Romani, J.-P., (2000). L'interlecte martiniquais, Approches sociolinguistiques des rapports langue-idéologie dans une communauté antillaise. Thèse de Doctorat en Sciences du Langage, Université de Rouen Haute Normandie, 2 tomes.

Romano, C. (2010). Au cœur de la raison, la phénoménologie, Paris, Folio Essais.

Riquois, E. (2014). Transposer le CECR dans les manuels de français langue étrangère ; une gageure? Le français dans le monde, Recherches et Applications, 55, 92 -105.

Robillard, D. (2011). Les vicissitudes et tribulations de "comprendre »: un enjeu en didactique des langues et des cultures? Dans P. Blanchet, P. Chardenet (dir.), Guide pour la recherche en didactique des langues et des cultures. Approches contextualisées, Paris, Editions des Archives contemporaines / AUF.

Vygotski, L. (1985). Pensée et Langage. Paris : Terrains / Éditions Sociales. 